\title{
Intra- und extrazelluläre Elektrolyt- und pH-Messungen vor und nach Hämodialyse
}

\author{
Von H. ZuMKLEY und E. SCHÜRMEYER \\ Aus der Medizinischen Klinik und Poliklinik der Universität Münster/Westf. \\ (Direktor: Prof. Dr. W. H. Hauss)
}

(Eingegangen am 12. Dezember 1967)

Herrn Prof Dr. W. H. Hauss zum 60. Geburtstag gewidmet

\begin{abstract}
Bei 6 Patienten mit chronischer Niereninsuffizienz wurden wiederholt vor und nach Hämodialyse Natrium und Kalium sowie aktuelles $\mathrm{pH}$, Standard-Bicarbonat, Basenexzess und aktuelles $\mathrm{HCO}_{3}^{-}$im Plasma und in den Erythrocyten bestimmt. Alle Patienten wiesen vor der Hämodialyse eine zum Teil erhebliche intra- und extrazelluläre Acidose sowie einen erniedrigten Kalium- und einen erhöhten Natriumquotienten auf. Nach der Hämodialyse zeigten sowohl die Kationenquotienten als auch die pH-Werte eine deutliche Tendenz zur Normalisierung auf. Es wird zu der Frage Stellung genommen, ob eine Beziehung zwischen den beobachteten Abweichungen der Kationenquotienten und der $\mathrm{pH}$-Werte besteht.

In 6 patients with chronic insufficiency of the kidneys, the levels of sodium and potassium, the actual $\mathrm{pH}$, standard bicarbonate, base excess, and actual $\mathrm{HCO}_{3}^{-}$were measured in the plasma and erythrocytes before and after haemodialysis. Before haemodialysis, all the patients showed an intra- and extracellular acidosis, which was sometimes very severe, and decreased potassium and increased sodium quotients. After haemodialysis, the cation quotients and the $\mathrm{pH}$ values showed a marked tendency to normalise. The possible relationship between the variation in the cation quotients and the $\mathrm{pH}$ values is discussed.
\end{abstract}

Veränderungen des Elektrolyt- und Säure-Basenhaushaltes gehören zur Symptomatik der chronischen Niereninsuffizienz. Durch einen planmäßigen Ausgleich dieser Veränderungen gelingt es oft, selbst schwere Urämien zu bessern. Voraussetzung hierfür ist jedoch eine exakte Analyse des Elektrolyt- und Säure-Basenhaushaltes. Bisher war man vornehmlich auf die Messung der leicht zugänglichen extrazellulären Veränderungen angewiesen.

In den letzten Jahren hat sich zunehmend die Erkenntnis durchgesetzt, daß die Analyse von Erythrocyten wichtige Aufschlüsse über das Verhalten der intrazellulären Elektrolyte zu geben vermag (1-5). Tierexperimentelle Untersuchungen lassen darauf schließen, daß die Elektrolytveränderungen in den Erythrocyten denjenigen anderer Zellsysteme parallel gehen (6-9). Auch das Verhalten des intrazellulären Säure-Basengleichgewichtes hat, seitdem die Möglichkeit einer Bestimmung in den Erythrocyten besteht, an klinischem Interesse zugenommen $(5,10,11)$. In einer früheren Arbeit konnten wir zeigen, daß es bei Patienten mit chronischer Niereninsuffizienz zu etheblichen Abweichungen der intrazellulären Natrium-, Kalium- und Magnesiumkonzentration kommt (2). Wir möchten jetzt über vergleichende Untersuchungen zwischen intra- und extrazellulärem Verhalten des Säure-Basenhaushaltes und der Natrium- und Kaliumquotienten bei Patienten mit chronischer Niereninsuffizienz vor und nach Hämodialyse berichten.

\section{Patientengut und Methodik}

Bei 6 Patienten mit chronischer Niereninsuffizienz wurden wiederholt vor und nach Hämodialyse Natrium und Kalium sowie aktuelles $\mathrm{pH}$, Standard-Bicarbonat, Basenexzess und akt. $\mathrm{HCO}_{3}^{-}$im Plasma und in den Erythrocyten bestimmt. Zur Beurteilung wurden die Kationenquotienten $\mathrm{Na}_{\mathrm{e}}^{+} / \mathrm{Na}_{\mathrm{i}}^{+}$und $\mathrm{K}_{\mathrm{i}}^{+} / \mathrm{K}_{\mathrm{e}}^{+}$herangezogen. Die Normalwerte beruhen auf Vergleichsuntersuchungen an 20
Gesunden. Die Bestimmung der intrazellulären Elektrolyte erfolgte an einem Exythrocytenkonzentrat, das durch $20 \mathrm{Min}$. langes Zentrifugieren mit 3000 U./Min. gewonnen wurde. (Einzelheiten siehe bei RIECKER (4) sowie LOSSE und Mitarbeiter $(13,14)$.

Die intra- und extrazellulären pH-Bestimmungen erfolgten nach der von GleichmaNN und Mitarbeiter (10) angegebenen Methode im Astrup-Mikroequipment der Fa. Radiometer, Kopenhagen. Die Messungen wurden in arteriell entnommenen Blutproben durchgeführt. Nach Bestimmung des extrazellulären Säure-Basenhaushaltes (akt. pH, Standard-Bicarbonat, Basenabweichung, $\mathrm{paO}_{2}, \mathrm{p}_{2} \mathrm{CO}_{2}$ ) wurde das Blut $10 \mathrm{Min}$. bei Zimmertemperatur und 4000 U./Min. unter Außerachtlassung anaerober Arbeitsbedingungen zentrifugiert. Nach Absaugen des Serums wurde das Erythrocytenkonzentrat in zwei Spritzen aufgenommen und durch mehrfaches Hindurchpressen durch einen verengten Zweiwegehahn mechanisch hämolysiert. Die Vollständigkeit der mechanischen Hämolyse wurde an Ausstrichen des Erythrocytenkonzentrates mikroskopisch kontrolliert. Anschließend wurde nach Äquilibrierung des Hämolysates mit 4 und $8 \% \mathrm{CO}_{2}$ in Sàuerstoff eine $\mathrm{pH} / \mathrm{log} \mathrm{pCO}_{2}$-Gerade aufgestellt. Der aktuelle $\mathrm{pH}$-Wert wurde indirekt bestimmt, indem mit dem extrazellulären $\mathrm{pCO}_{2}$ aus der $\mathrm{pH} / \mathrm{log} \mathrm{pCO}_{2}$-Geraden des Hämolysiates der zugehörige $\mathrm{pH}$-Wert aufgesucht wurde. Die intrazellulären Normwerte $(\mathrm{pH} 7,249 \pm$ 0,009; Standard-Bicarbonat 15,9 $\pm 0,4 \mathrm{mVal} / l$; Basenabweichung $-13,5 \pm 1,2 \mathrm{mVal} / \mathrm{l}$ ) wurden von GLEICHMANN und Mitarbeiter übernommen, nachdem orientierende Messungen zu übereinstimmenden Ergebnissen geführt hatten.

\section{Ergebnisse}

Abbildung 1 zeigt das Verhalten der intra- und extrazellulären $\mathrm{pH}$-Werte sowie des Natrium- und Kaliumquotienten bei Patienten mit chronischer Niereninsuffizienz vor und nach Hämodialyse. Bei àllen Patienten zeigt sich vor der Hämodialyse eine zum Teil erhebliche intra- und extrazelluläre Acidose. Gleichzeitig besteht ein erniedrigter Kaliumquotient sowie mit einer Ausnahme ein erhöhter Natriumquotient. Nach Hämodialyse zeigen sowohl die Kationenquotienten als auch die $\mathrm{pH}$ - Werte eine deutliche Tendenz zur Normalisierung. In den meisten Fällen wird ein normaler Wert erreicht. 


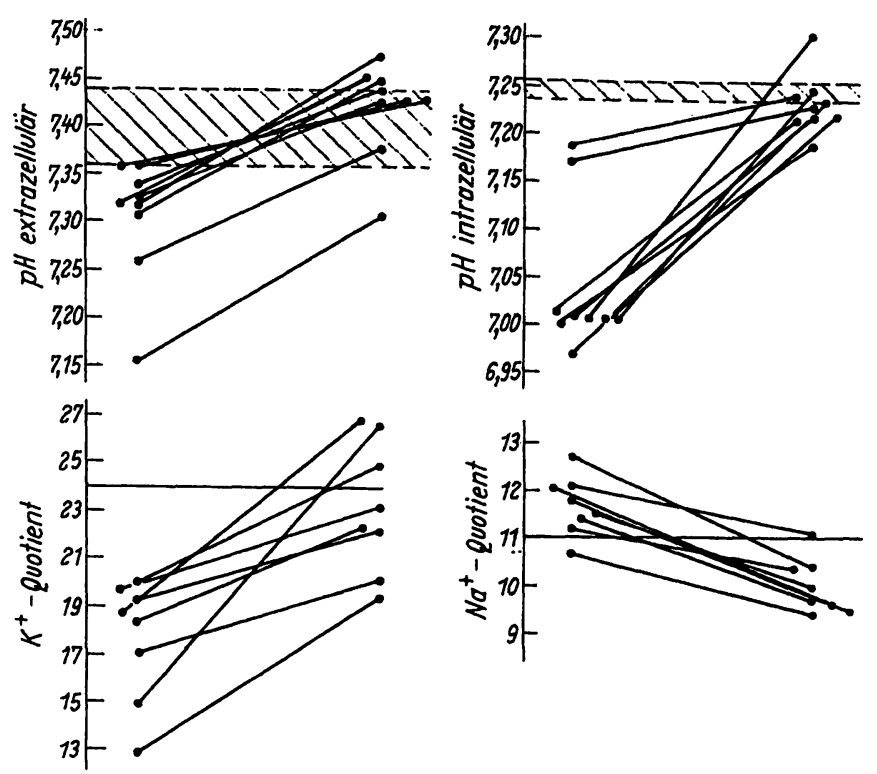

Abb. 1

Verhalten der intra- und extrazellulären pH-Werte sowie des Natriumund Kaliumquotienten bei Patienten mit chronischer Niereninsuffizienz vor und nach Hämodialyse

In Abbildung 2 sind in einem Diagramm die Kaliumquotienten gegen die intrazellulären (a) und extrazellulären (b) $\mathrm{pH}$-Werte aufgetragen. Es zeigt sich eine Abhängigkeit zwischen Änderungen des intraund extrazellulären $\mathrm{pH}$-Wertes und des Kaliumquotienten. Diese Abhängigkeit tritt besonders deutlich zwischen intrazellulärem $\mathrm{pH}$-Wert und Kaliumquotient hervor.

Abbildung 3 zeigt in einem Diagramm das Verhalten des Natriumquotienten zum intrazellulären (a) bzw. extrazellulären (b) $\mathrm{pH}$-Wert. Auch hier verlaufen die Änderungen des Natriumquotienten in deutlicher Abhängigkeit vom $\mathrm{pH}$-Wert.

\section{Besprechung der Ergebnisse}

Unsere Untersuchungen an Erythrocyten ergeben, daß bei Patienten mit chronischer Niereninsuffizienz die intra- und extrazellulären $\mathrm{pH}$-Werte zum Teil erheblich erniedrigt sind. Gleichzeitig besteht eine Verminderung -des Kalium- und eine Erhöhung des Natriumquotienten. Nach Hämodialyse zeigen alle Werte entweder eine deutliche Tendenz zur Normalisierung oder er- reichen den Normalbereich. Unsere Befunde weisen weiterhin eine deutliche Abhängigkeit der aufgeführten Kationenquotienten vom intra- und extrazellulären $\mathrm{pH}$-Wert auf.

Es wird heute allgemein angenommen, $\mathrm{da} B$ die hohe Kalium- und die niedrige Natrium-Konzentration in der Zelle durch einen Pumpmechanismus aufrecht erhalten wird, der entgegen den Konzentrationsgradienten $z$ wischen intra- und extrazellulären Raum, d. h. entgegen der passiven Diffusion ständig Kalium in die Zellen hinein und Natrium aus den Zellen heraus transportiert. Dieser aktive, energieabhängige Transport kann durch Schädigungen, die zu einer Hemmung der Glykolyse und Verminderung der energiereichen Phosphate in den Zellen führen, beeinträchtigt werden. Es kommt dann zu einem Ausstrom von Kalium und Einstrom von Natrium in die Zelle (15-17).

Veränderungen dieser Beziehungen zwischen extraund intrazellulärem Kationengehalt lassen sich durch Bildung der sogenannten Kationengradienten, wie wir es oben durchgeführt haben, gut charakterisieren. Nach Untersuchungen von GESSLER besteht eine gute Übereinstimmung zwischen Änderungen der Kationenquotienten an Erythrocyten verglichen mit tierexperimentellen Ergebnissen am Muskel (1, 2, 18, 19).

Wie bereits früher von uns diskutiert wurde, sind die bei Urämie auftretenden intrazellulären Elektrolytveränderungen Folge einer Hemmung des aktiven Transportmechanismus durch retinierte harnpflichtige Stoffe, wobei in erster Linie an die Anhäufung von Wasserstoffionen zu denken ist (12). Die aus unseren Untersuchungen hervorgehende Abhängigkeit zwischen intra- und extrazellulären $\mathrm{pH}$-Wert und Kalium- bzw. Natriumquotient spricht für eine derartige Annahme. Weiterhin lassen sich in diesem Zusammenhang tierexperimentelle Befunde anführen. So beobachtete GESSLER bei metabolischer Acidose eine Verminderung des muskulären Kaliumquotienten $(18,19)$. $\mathrm{Zu}$ gleichartigen Ergebnissen kam SCRIBNER bei respiratorischer Acidose (20). v. BubNoff und RIecker (21) sowie GleichmanN (10) wiesen ähnliche Veränderungen auch an Erythrocyten in vitro nach.

Bezüglich der beobachteten gleichsinnigen Veränderungen von $\mathrm{pH}$-Wert sowie Natrium- und Kalium-

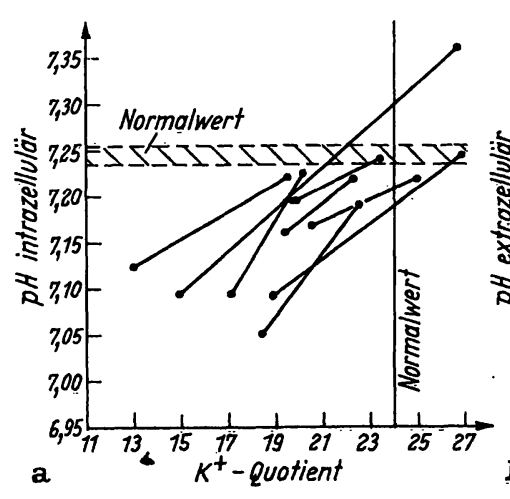

Abb. 2

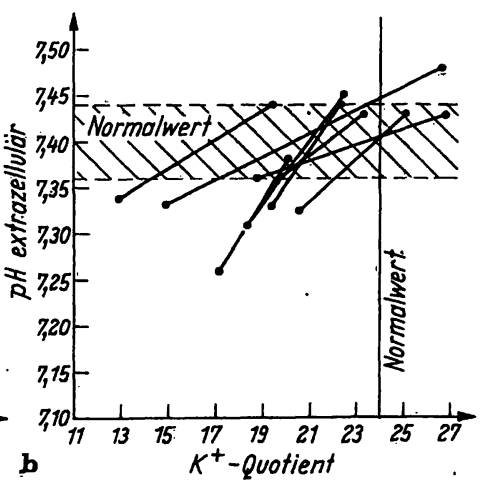

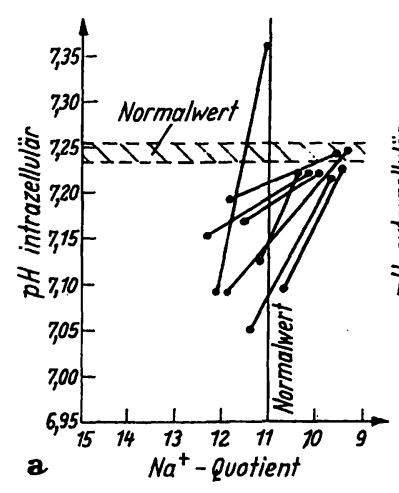

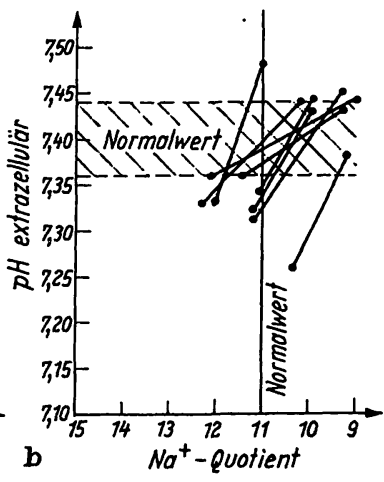

Abb. 3

Verhalten des Natriumquotienten gegenüber dem intrazellulären- (a)
Verhalten des Natriumquotienten gegenüber dem int

(a)

Verhalten des Kaliumquotienten gegenüber dem int
und extrazellulären (b) pH-Wert 
quotient ist weiterhin die Frage zu diskutieren, ob diese Abweichungen durch einen dritten unbekannten Faktor bei urämischen Patienten hervorgerufen werden (22). Diese Möglichkeit läßt sich durch unsere Befunde nicht sicher ausschließen, obgleich die oben aufgeführten experimentellen Untersuchungen eher daran denken lassen, daß die Änderungen des Natrium- und Kaliumquotienten durch den $\mathrm{pH}$-Wert beeinflußt werden.
Für die Behandlung der chronischen Niereninsuffizienz bzw. Urämie ergibt sich aus unseren Befunden, daß durch Einsatz der Hämodialyse sowohl Veränderungen des Säure-Basen- als auch des Elektrolythaushaltes behoben werden können. Diese Normalisierung ist wiederum die Voraussetzung dafür, $\mathrm{da} B$ weitere folgenschwere Störungen des Zellstoffwechsels vermieden werden bzw. eine normale Organfunktion wiederhergestellt wird.

\title{
Literatur
}

1. Gessler, U., Verh. Dtsch. Ges. inn. Med. 66, 870 (1960). 2.Gessler, U., Klin. Wschr. 39, 232 (1961). - 3. HäNZE, S., Klin. Wschr. 38, 769 (1960). - 4. RIECKER, G., Klin. Wschr. 35, 1158 (1957). - 5. RIECKER, G., Klin. Wschr. 41, 184 (1963). - 6. BURCK, H. C., Zschr. exper. Med. 144, 93 (1967). - 7. Wilbrandt, W., J. Pharmacy Pharmacol. London 11, 65 (1959). - 8. WILBRANDt, W., Klin. Wschr. 41, 138 (1963). - 9. ZuMKLEY, H., Zschr. Kreisl. forsch. 56, 678 (1967). - 10. GleichmanN, K., H. v. STUCKRAD und M. ZindLER, Zschr. exper. Med. 139, 255 (1965). 11. Sommerkamp, H. und K. Bomke, Klin. Wschr. 42, 392 (1964). 12. Zumklex, H. und H. Losse, Gastroenterologia Basel 104, 136 (1965). - 13. Losse, H., H. WehMeYer und F. WesseLs,
Klin. Wschr. 38, 393 (1960). - 14. Losse, H., H. ZUMKLEY und H. WeHMEYER, Zschr. Kreisl.forsch. 51, 752 (1962). - 15. BRESCIANI, F., F. Auricchio und C. Fiore: Nature, London 196, 186 (1962). - 16. Konsek, I. und C. Bishop, Proc. Soc. exp. Biol. Med. N.Y. 110, 813 (1962). - 17. WILBRAND̈r, W., Med. Klin. 50, 2085 (1962). - 18. Gessler, U. und W. Caliebe, Zschr. exper. Med. 134, 139 (1961). - 19. Gessier, U., Internist 3, 672 (1962). 20. Scribner, B. H., K. Fremont-Smith und J. M. Bưrnell, J. Clin. Invest. 34, 1276 (1955). - 21. Bubnoff, v. M. und G. RIECKER, Klin. Wschr. 39, 724 (1961). - 22. Sarre, H., U. Gessler und V. Heinze, Internist 10, 446 (1965).
Dr. H. Zumkley 44 Münster (Westf.) Westring 3

\section{Eine bewährte Methode zur routinemäßigen Bestimmung des proteingebundenen Jods (PBI)}

\author{
Von A. UETTWILLER \\ Aus den Laboratorien (Leiter: Dr. M. Keller) der Universitäts-Fraulenklinik Basel \\ (Direktor: Prof. Dr. Th. Koller)
}

(Eingegangen am 17. Dezember 1967)

\begin{abstract}
Es wird eine zuverlässige Routinemethode zur Bestimmung von PBI im Blutplasma beschrieben. Die oxydative Aufschlußzeit konnte wesentlich verkürzt werden. Eine Vereinfachung der Meßbedingungen wurde durch Unterbrechung der katalytischen Wirkung des Jods auf die Reaktion: $\mathrm{Ce}^{4+} \longrightarrow \mathrm{Ce}^{3+}$ mittels $\mathrm{Zusatz}$ von $\mathrm{Hg}^{2+}$-Ionen erreicht.
\end{abstract}

A reliable, routine method is described for the measurement of PBI in blood plasma. The time for oxidative cleavage was greatly reduced. The determination was simplified by adding $\mathrm{Hg}^{2+}$ ions, which stop the catalytic action of the iodine in the reaction: $\mathrm{Ce}^{4+} \longrightarrow \longrightarrow \mathrm{Ce}^{3+}$.

Es wird im folgenden eine Methode zur PBI-Bestimmung im Blutplasma beschrieben, die sowohl als Makro-' ( $1 \mathrm{~m} l$ Plasma) als auch Semimikro- $(200 \mu l)$ Methode in unserem Laboratorium seit einiger Zeit Anwendung findet. Das Verfahren basiert auf den Angaben von FARELL und Richmond (1) sowie denjenigen von FriedmanN (2), welche die nasse Veraschung von jodhaltigen Proteinen beschrieben.

Unsere wichtige Modifikation verkürzt die oxydative Aufschlußzeit von $4 \frac{1}{2}$ Stdn. auf $1 \mathrm{Std}$. Weiter werden die Meßbedingungen wesentlich vereinfacht, indem die katalytische Wirkung von $\mathrm{J}_{2}$ auf die Reaktion:

$$
\mathrm{Ce}^{+4}+\mathrm{As}^{+3} \longrightarrow \mathrm{Ce}^{+3}+\mathrm{As}^{+5}
$$

mit $\mathrm{Hg}^{+2}$-Ionen gestoppt wird. Die țechnischen Schwierigkeiten, welche die meisten bekannten Plasmajodbestimmungsmethoden aufweisen, konnten dadurch weitgehend behoben werden.

\section{Prinzip}

Das mit $\mathrm{HClO}_{4}$ gefällte Eiweiß wird mit einem Chlorsäure-Dichromat-Gemisch oxydativ aufgeschlossen:

$$
\mathrm{R}-\mathrm{J} \underset{\mathrm{HClO}_{2}}{\stackrel{\mathrm{K}_{2} \mathrm{Cr}_{2} \mathrm{O}_{7}}{\longrightarrow}} \mathrm{R}-+\mathrm{JO}_{\mathbf{3}}^{-}
$$

